\title{
Analysis of risk factors for hyponatremia in patients with acute spinal cord injury: a retrospective single-institution study in Japan
}

\author{
Hiroyuki Ohbe ${ }^{1} \cdot$ Tomoaki Koakutsu $^{2} \cdot$ Shigeki Kushimoto $^{2,3}$
}

Received: 27 June 2018 / Revised: 23 September 2018 / Accepted: 24 September 2018 / Published online: 16 October 2018

(c) International Spinal Cord Society 2018

\begin{abstract}
Study design: Retrospective single-institution observational study.

Objective: Spinal cord injury (SCI) is associated with a high prevalence of hyponatremia, which can cause various clinical symptoms and increase mortality. We aimed to analyze the risk factors for hyponatremia in patients with acute SCI.

Setting: Trauma Center, Tohoku University Hospital, Japan

Methods: We reviewed the medical records of patients with SCI admitted between January 2008 and November 2016. Least angle regression analyses was performed to assess the association between hyponatremia and other parameters.

Results: Of the 213 enrolled patients, $85(40 \%)$ had hyponatremia (serum $\mathrm{Na}<135 \mathrm{mmol} / \mathrm{L}$ ) whilst 19 (9\%) had severe hyponatremia (serum $\mathrm{Na}<130 \mathrm{mmol} / \mathrm{L}$ ). Least angle regression analyses revealed that hyponatremia was significantly associated with higher American Spinal Injury Association impairment scale, nosocomial pneumonia, deep vein thrombosis, bradycardia, and surgery for SCI before developing hyponatremia. Severe hyponatremia was significantly associated with traumatic brain injury with an abbreviated injury scale score $\geq 3$, higher American Spinal Injury Association impairment scale, bradycardia, vasopressors, and nosocomial pneumonia.

Conclusion: Various factors including traumatic brain injury with an abbreviated injury scale score $\geq 3$ and higher American Spinal Injury Association impairment scale may be associated with hyponatremia in patients with acute SCI. These factors can help clinicians identify and monitor SCI patients with a higher hyponatremia risk, and potentially help patients to avoid severe complications.
\end{abstract}

\section{Introduction}

Hyponatremia, defined as a serum sodium concentration of $<135 \mathrm{mmol} / \mathrm{L}$, is frequently encountered in clinical practice, occurring in $15-20 \%$ of patients following emergency admission and in up to $20 \%$ of critically ill patients $[1,2]$. Spinal cord injury (SCI) is associated with a higher prevalence of hyponatremia (25-80\%) within the first 2 weeks following trauma [3-6].

Hiroyuki Ohbe

hohbey@gmail.com

1 Department of Clinical Epidemiology and Health Economics, School of Public Health, The University of Tokyo, Tokyo, Japan

2 Department of Emergency and Critical Care Medicine/Emergency Center, Tohoku University Hospital, Sendai, Japan

3 Division of Emergency and Critical Care Medicine, Tohoku University Graduate School of Medicine, Sendai, Japan
Hyponatremia leads to various clinical symptoms that range from subtle to severe or life-threatening, and also leads to secondary SCI that is associated with increased mortality $[1-3,7,8]$. Therefore, early detection and management of hyponatremia is crucial for improving patient outcomes.

However, very few studies have focused on the risk factors for hyponatremia in SCI patients. Previous studies revealed potential causes of hyponatremia to be age, having a severe American Spinal Injury Association (ASIA) impairment scale grade, low blood pressure, the level of injury, and fluid overload [3-5, 9]. However, these studies were conducted in SCI centers and excluded patients with traumatic brain injury. The incidence rates of concomitant brain and spinal cord injuries have been reported to range from 25 to $60 \%[10,11]$. Therefore, these results cannot be generalized to patients in trauma centers, where patients with acute SCI are generally treated in most countries. In trauma centers, patients with acute SCI can have other potential causes of hyponatremia such as traumatic brain 
injury, having surgery for other traumas, excessive fluid intake, and nosocomial infections [10, 11].

In this study, we aimed to analyze the risk factors for hyponatremia in patients with acute SCI at a single tertiary emergency and critical care center in Japan.

\section{Methods}

\section{Patients}

In this retrospective observational study, we reviewed the charts of patients with acute SCI who were admitted to the Department of Emergency and Critical Care Medicine in Tohoku University Hospital between January 2008 and November 2016. Tohoku University Hospital is a 1225-bed Japanese, academic, tertiary care hospital. The emergency department accommodates approximately 3000 ambulance visits per year. Tertiary-level hospitals, which are authorized by the Japanese Ministry of Health, Labor and Welfare, are equivalent in terms of functionality to level 1 trauma centers in Europe and the United States. They are capable of providing 24-h specialty care in areas such as general surgery, cardiovascular surgery, orthopedic surgery, neurosurgery, anesthesiology, emergency medicine, radiology, internal medicine, and critical care [12]. Patients considered for this study were those admitted within $24 \mathrm{~h}$ after their initial trauma.

We included all patients with SCI or vertebral fracture during the study period. We excluded patients: (i) who were transferred to our hospital $\geq 2$ days after experiencing trauma; (ii) who were discharged or died within 3 days after experiencing trauma, (iii) who did not undergo more than two blood tests, including serum sodium concentration, during hospitalization; (iv) who were diagnosed as having simple transverse and spinous fracture with an ASIA impairment scale grade of E; [9] (v) who had a history of end-stage renal disease at admission; and (v) for whom information on outcome, abbreviated injury scale (AIS) score, or ASIA impairment scale grade was unavailable [9].

We defined "hyponatremia" as a serum sodium concentration $<135 \mathrm{mmol} / \mathrm{L}$ and "severe hyponatremia" as a serum sodium concentration $<130 \mathrm{mmol} / \mathrm{L}$ [1]. Patients undergoing hyponatremia treatment, i.e. receiving sodium supplementation, were considered to be patients with "severe hyponatremia" because treatment is not applicable when hyponatremia is mild, according to the clinical practice guidelines [1].

\section{Variables}

Patient charts were reviewed, focusing on age, sex and vital signs over the 24-h period following admission to the emergency department. Variables included Charlson comorbidity index (CCI); level of injury site; ASIA impairment scale grade at admission;[9] injury severity score (ISS); traumatic brain injury with an AIS score $\geq 3$; [13] the physiological findings within $24 \mathrm{~h}$ after trauma; any laboratory data during hospitalization and within 28 days after admission; diagnosis; surgery for SCI before developing hyponatremia; and interventional procedures during hospitalization (transfusion, respirator, vasopressors, steroids, and fluid infusion).

The decision regarding which clinical variables, including serum sodium concentration, would be tested was at the discretion of each clinician. If serum sodium concentration $<135 \mathrm{mmol} / \mathrm{L}$ was detected in patients within 28 days after admission, we classified them as having hyponatremia.

The CCI was calculated using the recorded diagnoses for each patient. Patients were categorized into four groups according to their $\mathrm{CCI}: \mathrm{CCI}=0, \mathrm{CCI}=1, \mathrm{CCI}=2$, and $\mathrm{CCI} \geq 3$ [14]. Patients were categorized into two groups according to their injury site: (i) cervical and (ii) thoracic and lumbar. The ASIA impairment scale is based on international standards for neurological classification of SCI [9]. To provide objective measurement, two skilled orthopedic surgeons judged the ASIA impairment scale grade at admission. Patients were categorized into three groups according to their ASIA impairment scale grade: A, B-D and E. The ISS is used to evaluate the severity of anatomic injuries. The injury diagnosis is recorded according to the AIS by using the AIS 90 Update 98 [13]. We defined multiple injury as having two or more body regions with AIS score $\geq 3$ [13]. We defined hypotension as systolic blood pressure $<90 \mathrm{mmHg}$ within $24 \mathrm{~h}$ after trauma and bradycardia as heart rate $<50 \mathrm{bpm}$ within $24 \mathrm{~h}$ after trauma. Cumulative fluid balance on day 1-2 was calculated as the cumulative fluid infusion on day 1-2 minus cumulative urine output on day 1-2.

\section{Statistical analysis}

Continuous variables are presented as median with interquartile range. Categorical variables are presented as the number of patients (percentage of the total). Number of days from injury to development of hyponatremia are presented as histogram of patients with hyponatremia. We performed univariate analysis to compare the characteristics between patients with severe hyponatremia $(\mathrm{Na}<130 \mathrm{mEq} /$ L) and those without hyponatremia, as well as between patients with hyponatremia $(\mathrm{Na}<135 \mathrm{mEq} / \mathrm{L})$ and those without hyponatremia, using chi-squared tests and Wilcoxon rank-sum tests as appropriate.

Least angle regression was performed to assess the association between potential predictors and the presence 
Table 1 Characteristics of patients with non-hyponatremia and severe hyponatremia

\begin{tabular}{|c|c|c|c|c|}
\hline Variables & $\begin{array}{l}\text { Total } \\
n=147\end{array}$ & $\begin{array}{l}\text { Non-hyponatremia } \\
n=128\end{array}$ & $\begin{array}{l}\text { Severe hyponatremia } \\
n=19\end{array}$ & $P$-value \\
\hline Age, year & $58(37,72)$ & $58(35,72)$ & $63(60,77)$ & 0.034 \\
\hline Male & $115(78 \%)$ & $98(77 \%)$ & $17(89 \%)$ & 0.25 \\
\hline \multicolumn{5}{|l|}{$\mathrm{CCI}$ original } \\
\hline 0 & $105(71 \%)$ & $92(72 \%)$ & $13(68 \%)$ & \multirow[t]{4}{*}{0.49} \\
\hline 1 & $25(17 \%)$ & $22(17 \%)$ & $3(16 \%)$ & \\
\hline 2 & $11(8 \%)$ & $8(6 \%)$ & $3(16 \%)$ & \\
\hline $3-$ & $6(4 \%)$ & $6(5 \%)$ & $0(0 \%)$ & \\
\hline \multicolumn{5}{|l|}{ Injured site } \\
\hline Cervical & $86(59 \%)$ & $72(56 \%)$ & $14(74 \%)$ & \multirow[t]{2}{*}{0.21} \\
\hline Thoracic-lumbar & $61(41 \%)$ & $56(44 \%)$ & $5(26 \%)$ & \\
\hline \multicolumn{5}{|l|}{ ASIA impairment scale } \\
\hline A & $22(15 \%)$ & $15(12 \%)$ & $7(37 \%)$ & \multirow[t]{3}{*}{0.011} \\
\hline B-D & $51(35 \%)$ & $44(34 \%)$ & $7(37 \%)$ & \\
\hline $\mathrm{E}$ & $74(50 \%)$ & $69(54 \%)$ & $5(26 \%)$ & \\
\hline Injury severity score & $17(13,25)$ & $17(13,25)$ & $25(16,25)$ & 0.12 \\
\hline TBI with an AIS score $\geq 3$ & $58(40 \%)$ & $47(37 \%)$ & $11(58 \%)$ & 0.085 \\
\hline Multiple trauma & $82(56 \%)$ & $74(58 \%)$ & $8(42 \%)$ & 0.22 \\
\hline Hypotension & $37(25 \%)$ & $30(23 \%)$ & $7(37 \%)$ & 0.26 \\
\hline Bradycardia & $6(4 \%)$ & $3(2 \%)$ & $3(16 \%)$ & 0.029 \\
\hline \multicolumn{5}{|l|}{ Treatment } \\
\hline $\begin{array}{l}\text { Surgery for SCI before } \\
\text { developing hyponatremia }\end{array}$ & $85(58 \%)$ & $69(54 \%)$ & $16(84 \%)$ & 0.013 \\
\hline Transfusion & $33(22 \%)$ & $27(21 \%)$ & $6(32 \%)$ & 0.38 \\
\hline Mechanical ventilation & $42(29 \%)$ & $33(26 \%)$ & $9(47 \%)$ & 0.061 \\
\hline Vasopressors & $29(20 \%)$ & $21(16 \%)$ & $8(42 \%)$ & 0.014 \\
\hline Steroids & $28(19 \%)$ & $24(19 \%)$ & $4(21 \%)$ & 0.76 \\
\hline $\begin{array}{l}\text { Cumulative fluid infusion on day } \\
1-2, \mathrm{~mL}\end{array}$ & $\begin{array}{l}4920(3628, \\
7521)\end{array}$ & $4800(3293,7334)$ & $6386(4446,8496)$ & 0.060 \\
\hline $\begin{array}{l}\text { Cumulative fluid balance on days } \\
1-2, \mathrm{~mL}\end{array}$ & $\begin{array}{l}1042(-61 \\
3594)\end{array}$ & $930(-172,3668)$ & $1551(306,3520)$ & 0.28 \\
\hline \multicolumn{5}{|l|}{ Outcome } \\
\hline Nosocomial pneumonia & $29(20 \%)$ & $20(16 \%)$ & $9(11 \%)$ & 0.003 \\
\hline Deep vein thrombosis & $5(3 \%)$ & $4(3 \%)$ & $1(1 \%)$ & 0.50 \\
\hline
\end{tabular}

Binary variables are presented as number of patients (percentage of the total), and continuous variables are presented as median (25th, 75th percentile)

Binary variables are compared using chi-square tests and continuous variables are compared using Wilcoxon rank-sum tests

CCI Charlson Comorbidity Index, ASIA American Spinal Injury Association, TBI traumatic brain injury, AIS abbreviated injury scale, $S C I$ spinal cord injury of severe hyponatremia as the primary analysis, and hyponatremia as the secondary analysis $[15,16]$. Least angle regression is a method of model-building that shrinks the coefficients by making the sum of the squared coefficients less than a constant. This results in less bias in the estimated coefficients, and any bias present is toward zero rather than away from zero $[15,16]$. To perform least angle regression, we used "LARS: STATA module to perform least angle regression" which was provided by Adrian [17]. The variables presented in Table 1 and Table 3 were used in the least angle regression model. A threshold for significance was $P<0.05$. All statistical analyses were conducted using IBM SPSS version 25.0 (IBM Corporation, Armonk, NY, USA) and STATA/MP 14.2 (StataCorp, College Station, TX, USA). 


\section{Results}

The records yielded 507 patients with acute SCI or vertebral fracture. Two hundred thirteen patients were included in the study after application of the inclusion and exclusion criteria (Fig. 1). Of the 213 enrolled patients, $19(9 \%)$ had severe hyponatremia (serum $\mathrm{Na}<130 \mathrm{mmol} / \mathrm{L}$ ) and 85 (40\%) had hyponatremia (serum $\mathrm{Na}<135 \mathrm{mmol} / \mathrm{L}$ ). Median (interquartile range) frequency of the serum sodium tests during first 7 days and 14 days were 5 tests (4-6) and 8 tests (6-11), respectively.

Figure 2 shows the number of days from injury to the development of hyponatremia in patients with hyponatremia. Median (interquartile) of number of days from injury to developing hyponatremia were 7 (4-11).

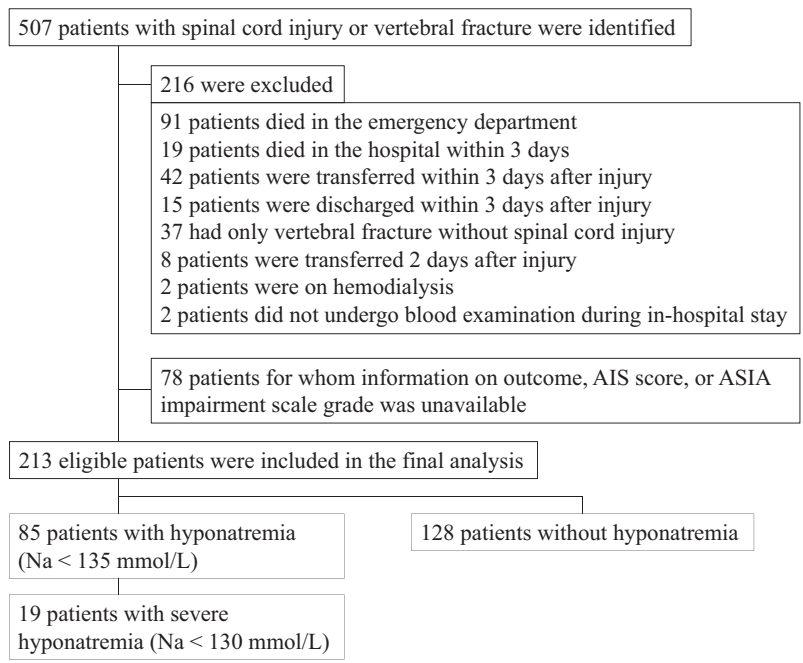

Fig. 1 Flow diagram of patient selection AIS abbreviated injury scale, ASIA American Spinal Injury Association

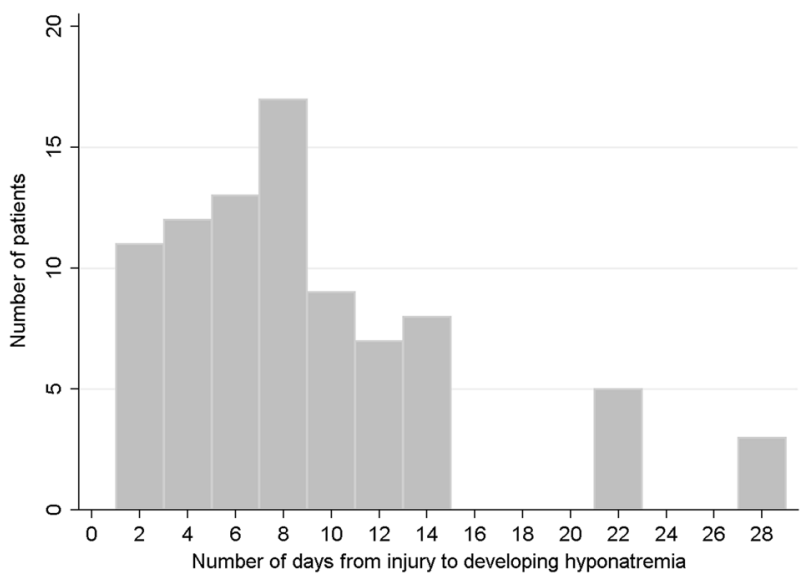

Fig. 2 Number of days from injury to the development of hyponatremia presented as a histogram of patients with hyponatremia
Characteristics of patients with or without severe hyponatremia are shown in Table 1. Table 2 shows the association between severe hyponatremia and other variables according to least angle regression analyses. Nosocomial pneumonia, higher ASIA impairment scale, bradycardia, vasopressors TBI with an AIS score $\geq 3$, and age were found to be important covariates associating with severe hyponatremia.

Patient characteristics in patients with or without hyponatremia are shown in Table 3. Table 4 shows the association between hyponatremia and other variables according to the least angle regression analyses. Nosocomial pneumonia, deep vein thrombosis, bradycardia, surgery for SCI before developing hyponatremia, and higher ASIA impairment scale were found to be important covariates associating with hyponatremia.

\section{Discussion}

We assessed risk factors for hyponatremia in trauma patients with acute SCI at a single tertiary emergency and critical care center in Japan. In patients with acute SCI, we found associations between severe hyponatremia and various risk factors including ASIA impairment scale and TBI with an AIS score $\geq 3$.

The prevalence rate of hyponatremia (serum $\mathrm{Na}<135$ $\mathrm{mmol} / \mathrm{L}$ ) following SCI is reported to be high at $\sim 25-80 \%$ [3-6], but patients with hyponatremia are usually asymptomatic and do not require treatment $[1,2]$. In contrast, patients with severe hyponatremia (serum $\mathrm{Na}<130 \mathrm{mmol} /$ L) usually display various signs and symptoms, and are associated with a substantially higher mortality rate $[2,18,19]$. The prevalence rate of severe hyponatremia (serum $\mathrm{Na}<130 \mathrm{mmol} / \mathrm{L}$ ) in our cohort $(9 \%)$ was higher

Table 2 Results from least angle regression analyses of the association between potential predictors and the presence of severe hyponatremia

\begin{tabular}{|c|c|c|}
\hline Step Cp & $R^{2} \quad$ Action & $\begin{array}{l}\text { Odds ratio in final } \\
\text { model }\end{array}$ \\
\hline
\end{tabular}

\begin{tabular}{|c|c|c|c|c|}
\hline 1 & 18.24 & 0.00 & Intercept & \\
\hline 2 & 18.35 & 0.01 & + nosocomial pneumonia & 1.11 \\
\hline 3 & 17.27 & 0.03 & $\begin{array}{l}\text { +higher ASIA impairment } \\
\text { scale }\end{array}$ & 1.06 \\
\hline 4 & 9.90 & 0.09 & +bradycardia & 1.18 \\
\hline 5 & 11.58 & 0.09 & + TBI with an AIS score $\geq 3$ & 1.05 \\
\hline 6 & 8.98 & 0.12 & +vasopressors & 1.03 \\
\hline 7 & 6.63 & 0.15 & + age & 1.00 \\
\hline
\end{tabular}

$\mathrm{Cp}=$ Estimate of prediction error (minimum printed in bold)

ASIA American Spinal Injury Association, TBI traumatic brain injury, $A I S$ abbreviated injury scale 
Table 3 Characteristics of patients with non-hyponatremia and hyponatremia

\begin{tabular}{|c|c|c|c|c|}
\hline Variables & $\begin{array}{l}\text { Total } \\
n=213\end{array}$ & $\begin{array}{l}\text { Non-hyponatremia } \\
n=128\end{array}$ & $\begin{array}{l}\text { Hyponatremia } \\
n=85\end{array}$ & P-value \\
\hline Age, year & $58(36,72)$ & $58(35,72)$ & $60(37,73)$ & 0.29 \\
\hline Male & $167(78 \%)$ & $98(77 \%)$ & $69(81 \%)$ & 0.5 \\
\hline \multicolumn{5}{|l|}{$\mathrm{CCI}$ original } \\
\hline 0 & $149(70 \%)$ & $92(72 \%)$ & $57(67 \%)$ & \multirow[t]{4}{*}{0.34} \\
\hline 1 & $41(19 \%)$ & $22(17 \%)$ & $19(22 \%)$ & \\
\hline 2 & $16(8 \%)$ & $8(6 \%)$ & $8(9 \%)$ & \\
\hline $3-$ & $7(3 \%)$ & $6(5 \%)$ & $1(1 \%)$ & \\
\hline \multicolumn{5}{|l|}{ Injured site } \\
\hline Cervical & $122(57 \%)$ & $72(56 \%)$ & $50(59 \%)$ & \multirow[t]{2}{*}{0.78} \\
\hline Thoracic-lumbar & $91(43 \%)$ & $56(44 \%)$ & $35(41 \%)$ & \\
\hline \multicolumn{5}{|l|}{ ASIA impairment scale } \\
\hline A & $34(16 \%)$ & $15(12 \%)$ & $19(22 \%)$ & \multirow[t]{3}{*}{0.11} \\
\hline B-D & $72(34 \%)$ & $44(34 \%)$ & $28(33 \%)$ & \\
\hline $\mathrm{E}$ & $107(50 \%)$ & $69(54 \%)$ & $38(45 \%)$ & \\
\hline Injury Severity Score & $20(13,29)$ & $17(13,25)$ & $22(16,29)$ & 0.02 \\
\hline TBI with an AIS score $\geq 3$ & $83(39 \%)$ & $47(37 \%)$ & $36(42 \%)$ & 0.47 \\
\hline Multiple trauma & $125(59 \%)$ & $74(58 \%)$ & $51(60 \%)$ & 0.78 \\
\hline Hypotension & $54(25 \%)$ & $30(23 \%)$ & $24(28 \%)$ & 0.52 \\
\hline Bradycardia & $10(5 \%)$ & $3(2 \%)$ & $6(7 \%)$ & 0.093 \\
\hline \multicolumn{5}{|l|}{ Treatment } \\
\hline $\begin{array}{l}\text { Surgery for SCI before developing } \\
\text { hyponatremia }\end{array}$ & $105(49 \%)$ & $69(54 \%)$ & $36(42 \%)$ & 0.12 \\
\hline Transfusion & $51(24 \%)$ & $27(21 \%)$ & $24(28 \%)$ & 0.25 \\
\hline Mechanical ventilation & $63(30 \%)$ & $33(26 \%)$ & $30(35 \%)$ & 0.17 \\
\hline Vasopressors & $43(20 \%)$ & $21(16 \%)$ & $22(26 \%)$ & 0.12 \\
\hline Steroids & $40(19 \%)$ & $24(19 \%)$ & $16(19 \%)$ & 1 \\
\hline $\begin{array}{l}\text { Cumulative fluid infusion on day } \\
1-2, \mathrm{~mL}\end{array}$ & $\begin{array}{l}5401(3834, \\
7808)\end{array}$ & $4800(3293,7334)$ & $6421(4630,8270)$ & 0.057 \\
\hline $\begin{array}{l}\text { Cumulative fluid balance on days } \\
1-2, \mathrm{~mL}\end{array}$ & $\begin{array}{l}1541(88 \\
3816)\end{array}$ & $930(-172,3668)$ & $2335(621,4407)$ & 0.018 \\
\hline \multicolumn{5}{|l|}{ Outcome } \\
\hline Nosocomial pneumonia & $51(24 \%)$ & $20(16 \%)$ & $31(36 \%)$ & $<0.001$ \\
\hline Deep vein thrombosis & $13(6 \%)$ & $4(3 \%)$ & $9(11 \%)$ & 0.038 \\
\hline
\end{tabular}

Binary variables are presented as number of patients (percentage of the total), and continuous variables are presented as median (25th, 75th percentile)

Binary variables are compared using chi-square tests and continuous variables are compared using Wilcoxon rank-sum tests

CCI Charlson Comorbidity Index, ASIA American Spinal Injury Association, TBI traumatic brain injury, AIS abbreviated injury scale, $S C I$ spinal cord injury than that in patients admitted to the intensive care unit in a previous study (3.9\%) [2].

Hyponatremia was significantly associated with a higher ASIA impairment grade. This finding is consistent with that of previous studies [5]. The pathophysiological mechanism of hyponatremia in patients with SCI remains unclear. One previous postmortem study indicated that hyponatremia in patients with SCI was associated with disrupted integrity of descending renal sympathetic circuits that mediate the renin-angiotensin response to neural injury [20]. The primary physiological mechanism of hyponatremia might be explained by the syndrome of the inappropriate antidiuresis (SIADH) model or the renal sodium loss model (renal salt wasting) $[6,21]$; however, we were unable to identify the physiological mechanism as we did not investigate changes in urinary electrolytes or hormones.

To our knowledge, our study is the first to show that severe traumatic brain injury is related to severe 
Table 4 Results from least angle regression analyses of the association between potential predictors and the presence of hyponatremia

\begin{tabular}{|c|c|c|c|c|}
\hline Step & $\mathrm{Cp}$ & $R^{2}$ & Action & $\begin{array}{l}\text { Odds ratio in } \\
\text { final model }\end{array}$ \\
\hline 1 & 12.88 & 0.00 & Intercept & \\
\hline 2 & 6.15 & 0.04 & + nosocomial pneumonia & 1.23 \\
\hline 3 & 6.79 & 0.05 & + deep vein thrombosis & 1.18 \\
\hline 4 & 7.63 & 0.05 & +bradycardia & 1.17 \\
\hline 5 & 9.20 & 0.05 & $\begin{array}{l}\text { +surgery for SCI before } \\
\text { developing hyponatremia }\end{array}$ & 0.91 \\
\hline 6 & 2.47 & 0.09 & $\begin{array}{l}\text { +higher ASIA impairment } \\
\text { scale }\end{array}$ & 1.05 \\
\hline 7 & 4.02 & 0.09 & + TBI with an AIS score $\geq 3$ & 1.03 \\
\hline 8 & 2.00 & 0.11 & $\begin{array}{l}+ \text { cumulative fluid infusion on } \\
\text { day } 1-2\end{array}$ & 1.00 \\
\hline
\end{tabular}

$\mathrm{Cp}=$ Estimate of prediction error (minimum printed in bold)

SCI spinal cord injury, ASIA American Spinal Injury Association, TBI traumatic brain injury, $A I S$ abbreviated injury scale

hyponatremia in acute SCI patients. Traumatic brain injury are well known causes of hyponatremia [21-23]. Our study also suggested that combination of severe traumatic brain injury and acute SCI might cause severe hyponatremia.

Our study has some limitations. First, we did not measure the doses used for therapeutic sodium supplementation. Second, our exclusion criteria, which included lack of information on outcome, AIS score, or ASIA impairment scale grade, may result in selection bias. Third, we did not measure urine electrolyte and hormone levels in these patients because our primary goal was to assess risk factors rather than the pathophysiology of hyponatremia in patients with acute SCI. Therefore, we cannot speculate regarding the mechanism underlying the development of hyponatremia after SCI. Further studies are needed to elucidate this mechanism. Fourth, we did not assess pseudo-hyponatremia, medication-induced hyponatremia and dilutional hyponatremia, because most of the patients who developed hyponatremia did not measure specific factors relating to hyponatremia such as serum blood sugar, serum triglycerides, serum osmotic pressure, urine osmotic pressure, and urine electrolytes in our retrospective database. Fifth, because of the small sample size in this study, volumes of fluid infusions may not have shown a significant relationship with hyponatremia.

In conclusion, our study suggests that various factors including ASIA impairment scale and TBI with an AIS score $\geq 3$ may be associated with severe hyponatremia in patients with acute SCI. Clinicians should closely monitor the sodium levels of acute SCI patients with these risk factors and implement intensive sodium supplementation when appropriate to help patients avoid severe complications.

Author contributions $\mathrm{HO}$ and SK designed the study; HO and TK conducted the research; HO and TK analyzed the data; HO, TK, and SK wrote the paper; HO had primary responsibility for final content. All authors read and approved the final manuscript.

\section{Compliance with ethical standards}

Statement of ethics This study was approved by the Institutional Review Board of Tohoku University Hospital.

Conflict of interest The authors declare that they have no conflict of interest.

\section{References}

1. Spasovski G, Vanholder R, Allolio B, Annane D, Ball S, Bichet $\mathrm{D}$, et al. Clinical practice guideline on diagnosis and treatment of hyponatraemia. Intensive Care Med. 2014;40:320-31.

2. Funk GC, Lindner G, Druml W, Metnitz B, Schwarz C, Bauer P, et al. Incidence and prognosis of dysnatremias present on ICU admission. Intensive Care Med. 2010;36:304-11.

3. Biyani A, Inman CG, el Masry WS. Hyponatraemia after acute spinal injury. Injury. 1993;24:671-3.

4. Peruzzi WT, Shapiro BA, Meyer PR, Krumlovsky F, Seo BW. Hyponatremia in acute spinal cord injury. Crit Care Med. 1994;22:252-8.

5. Nakao Y, Suda K, Shimokawa N, Fu Y. Risk factor analysis for low blood pressure and hyponatremia in acutely and subacutely spinal cord injured patients. Spinal Cord. 2012;50:285-8.

6. Kriz J, Schuck O, Horackova M. Hyponatremia in spinal cord injury patients: new insight into differentiating between the dilution and depletion forms. Spinal Cord. 2015;53:291-6.

7. Upadhyay A, Jaber BL, Madias NE. Epidemiology of hyponatremia. Semin Nephrol. 2009;29:227-38.

8. Teasell RW, Arnold JM, Krassioukov A, Delaney GA. Cardiovascular consequences of loss of supraspinal control of the sympathetic nervous system after spinal cord injury. Arch Phys Med Rehabil. 2000;81:506-16.

9. Kirshblum S, Waring W 3rd. Updates for the International Standards for Neurological Classification of Spinal Cord Injury. Phys Med Rehabil Clin N Am. 2014;25:505-17.

10. Kushner DS, Alvarez G. Dual diagnosis: traumatic brain injury with spinal cord injury. Phys Med Rehabil Clin N Am. 2014;25:681-96.

11. Bourassa-Moreau E, Mac-Thiong JM, Ehrmann Feldman D, Thompson C, Parent S. Complications in acute phase hospitalization of traumatic spinal cord injury: does surgical timing matter? J Trauma Acute Care Surg. 2013;74:849-54.

12. The Ministry of Health, Labour and Welfare in Japan: On the current state and problems of emergency medical system. 13 Jan 2018. Available at: https://www.mhlw.go.jp/content/10802000/ 000328610.pdf. Accessed 10 October 2018.

13. Petrucelli E, States JD, Hames LN. The abbreviated injury scale: evolution, usage and future adaptability. Accid Anal Prev. 1981;13:29-35.

14. Quan H, Li B, Couris CM, Fushimi K, Graham P, Hider P. Updating and validating the Charlson comorbidity index and score for risk adjustment in hospital discharge abstracts using data from 6 countries. Am J Epidemiol. 2011;173:676-82. 
15. Tibshirani R. Regression shrinkage and selection via the lasso. J R Stat Soc B. 1996;58:267-88.

16. Efron B, Hastie T, Johnstone I, Tibshirani R. "Least angle regression" (with discussion) annals of statistics. 2003 [cited 8 Dec 2010]. Available at: http://www-stat.stanford.edu/ hastie/Pa pers/LARS/LeastAngle_2002.pdf

17. Adrian M. "LARS: Stata module to perform least angle regression," Statistical Software Components S456860, Boston College Department of Economics, 2006 (revised 22 Jul 2014).

18. Moore K, Thompson C, Trainer P. Disorders of water balance. Clin Med. 2003;3:28-33.

19. Patel GP, Balk RA. Recognition and treatment of hyponatremia in acutely ill hospitalized patients. Clin Ther. 2007;29:211-29.
20. Furlan JC, Fehlings MG, Shannon P, Norenberg MD, Krassioukov AV. Descending vasomotor pathways in humans: correlation between axonal preservation and cardiovascular dysfunction after spinal cord injury. J Neurotrauma. 2003;20:1351-63.

21. Spasovski G, Vanholder R, Allolio B, Annane D, Ball S, Bichet $\mathrm{D}$, et al. Clinical practice guideline on diagnosis and treatment of hyponatraemia. Nephrol Dial Transplant. 2014;29:i1-i39.

22. Rajagopal R, Swaminathan G, Nair S, Joseph M. Hyponatremia in traumatic brain injury: a practical management protocol. World Neurosurg. 2017;108:529-33.

23. Yumoto T, Sato K, Ugawa T, Ichiba S, Ujike Y. Prevalence, risk factors, and short-term consequences of traumatic brain injuryassociated hyponatremia. Acta Med Okayama. 2015;69:213-8. 